\title{
Inhibition of Rho-kinase is involved in the therapeutic effects of atorvastatin in heart ischemia/reperfusion
}

\author{
CHAO CHENG $^{1 *}$, XIAO-BO LIU ${ }^{2 *}$, SHAO-JIE BI ${ }^{1}$, QING-HUA LU ${ }^{1}$ and JUAN ZHANG ${ }^{1}$ \\ ${ }^{1}$ Department of Cardiology, The Second Hospital, Cheeloo College of Medicine, Shandong University, \\ Jinan, Shandong 250033; ${ }^{2}$ Shandong Blood Center, Jinan, Shandong 250012, P.R. China
}

Received July 19, 2019; Accepted March 24, 2020

DOI:10.3892/etm.2020.9070

\begin{abstract}
The aim of the present study was to investigate the effects of atorvastatin against heart ischemia/reperfusion (I/R) injury and its potential underlying mechanism. Rats were allocated into the following groups: Sham, I/R, atorvastatin $(10 \mathrm{mg} / \mathrm{kg}$ daily), fasudil $(10 \mathrm{mg} / \mathrm{kg}$ daily $)$ and atorvastatin + fasudil in combination. Drugs were administered for 2 weeks prior to I/R injury. I/R was established by ligating the left anterior descending branch (LAD) for $30 \mathrm{~min}$ and releasing the ligature for $180 \mathrm{~min}$. The I/R group was found to have increased myocardial infarct size, cardiomyocyte apoptosis, levels of plasma interleukin (IL)-6 and tumor necrosis factor (TNF)- $\alpha$, superoxide dismutase (SOD) activity, malondialdehyde (MDA) levels and Rho-kinase activity compared with the other treatment groups $(\mathrm{P}<0.05)$. Moreover, pretreatment with atorvastatin significantly attenuated Rho-kinase activity, myocardial infarct size, cardiomyocyte apoptosis, levels of plasma IL- 6 and TNF- $\alpha$, SOD activity and MDA levels, and upregulated nitric oxide production. It was also indicated that the specific Rho-kinase inhibitor, fasudil, had the same effects as atorvastatin in I/R. Therefore, the present results suggested atorvastatin may lead to cardiovascular protection, which may be mediated by Rho-kinase inhibition in heart I/R injury.
\end{abstract}

\section{Introduction}

Ischemic heart disease is a leading cause of mortality worldwide (1). Furthermore, acute myocardial infarction (AMI) is the most severe type of ischemic heart disease and has a high mortality rate (2). Timely reperfusion remains critical for

Correspondence to: Dr Juan Zhang, Department of Cardiology, The Second Hospital, Cheeloo College of Medicine, Shandong University, 247 Beiyuan Road, Jinan, Shandong 250033, P.R. China

E-mail: awwa6940@sina.com

${ }^{*}$ Contributed equally

Key words: Rho-kinase activity, heart ischemia/reperfusion, atorvastatin, fasudil the treatment of AMI; however, reperfusion may exacerbate metabolic dysfunction and structural damage to the myocardium, which is known as myocardial ischemia/reperfusion (I/R) injury (3). Myocardial I/R is characterized by endothelial dysfunction, cellular calcium overload, oxidative stress, inflammatory response and myocyte death, which all amplify tissue injury (4). Therefore, alleviating $I / R$ injury during myocardial reperfusion represents a crucial clinical challenge.

The ubiquitously expressed Rho-kinase, which is a serine/threonine kinase, has been reported to serve an important role in a number of major cardiovascular diseases, such as hypertension, heart failure, pulmonary hypertension, atherosclerosis, myocardial infarction and myocardial I/R (5-11). Previous studies have demonstrated that Rho-kinase activation is involved in the pathogenesis of $\mathrm{I} / \mathrm{R}$ in vivo (12-16). It has also been shown that fasudil, a Rho-kinase inhibitor, inhibits the activation of Rho-kinase during $\mathrm{I} / \mathrm{R}$ and reduces infarct size and myocyte apoptosis in rats $(17,18)$. Moreover, there has been increasing research into Rho-kinase as a potential therapeutic target in myocardial I/R.

Statins, which are competitive inhibitors of 3-hydroxy-3methylglutaryl coenzyme A (HMG-CoA) reductase, are used for treatment of dyslipidemias and prevention of cardiovascular diseases (19), as they decrease cholesterol biosynthesis, reduce low-density lipoprotein (LDL) cholesterol and triglyceride levels, and increase levels of high-density lipoprotein cholesterol (20). Previous studies have also reported that statins may exert cardiovascular protective effects and may inhibit Rho-kinase activity independent of LDL reduction, which are referred to as pleiotropic effects (21-25). However, the effect of statins on Rho-kinase activity in heart I/R injury in vivo requires further investigation. Therefore, it was hypothesized that cardioprotection of statins may be associated with inhibition of Rho-kinase in myocardial I/R injury.

\section{Materials and methods}

Animals. Female Wistar rats (age, 130 days; weight, 250-300 g) were obtained from Shandong University and acclimatized for 1 week prior to any experimentation. Rats were allowed free access to water and standard chow diet. All animals were housed under the following conditions: Standard lighting at 12:12 $\mathrm{h}$ light-dark cycle, temperature, $22 \pm 0.5^{\circ} \mathrm{C}$ and humidity, $60 \pm 10 \%$. The present study was approved by the Ethics 
Table I. Serum levels of IL-6, TNF- $\alpha$ and NO.

\begin{tabular}{lcccrr}
\hline Variables & Sham & I/R & I/R + A & I/R + F & I/R + A + F \\
\hline IL-6, pg/ml & $97.5 \pm 31.36$ & $375.1 \pm 140.39^{\mathrm{a}}$ & $261.5 \pm 81.28^{\mathrm{a}, \mathrm{b}}$ & $241.5 \pm 62.61^{\mathrm{a}, \mathrm{b}}$ & $224.9 \pm 55.77^{\mathrm{a}, \mathrm{b}}$ \\
TNF- $\alpha, \mathrm{pg} / \mathrm{ml}$ & $76.2 \pm 32.55$ & $545.5 \pm 161.27^{\mathrm{a}}$ & $322.1 \pm 59.68^{\mathrm{a}, \mathrm{b}}$ & $331.3 \pm 66.54^{\mathrm{a}, \mathrm{b}}$ & $300.875 \pm 71.42^{\mathrm{a}, \mathrm{b}}$ \\
$\mathrm{NO}, \mu \mathrm{mol} / \mathrm{l}$ & $7.83 \pm 1.91$ & $4.56 \pm 1.25^{\mathrm{a}}$ & $9.55 \pm 2.26^{\mathrm{b}}$ & $9.17 \pm 1.96^{\mathrm{b}}$ & $10.40 \pm 2.39^{\mathrm{b}}$ \\
\hline
\end{tabular}

$\mathrm{N}=12$, each group. ${ }^{\text {P }}<0.05$ vs. sham group. ${ }^{\mathrm{b}} \mathrm{P}<0.05$ vs. I/R group. $\mathrm{I} / \mathrm{R}$ group. $\mathrm{I} / \mathrm{R}$, ischemia/reperfusion; $\mathrm{I} / \mathrm{R}+\mathrm{A}, \mathrm{I} / \mathrm{R}+$ atorvastatin; $\mathrm{I} / \mathrm{R}+\mathrm{F}$, $\mathrm{I} / \mathrm{R}+$ fasudil; I/R + A + F, I/R + atorvastatin + fasudil; IL, interleukin; TNF, tumor necrosis factor; NO, nitric oxide.

Table II. SOD activity and MDA levels.

\begin{tabular}{|c|c|c|c|c|c|}
\hline Variables & Sham & $\mathrm{I} / \mathrm{R}$ & $\mathrm{I} / \mathrm{R}+\mathrm{A}$ & $\mathrm{I} / \mathrm{R}+\mathrm{F}$ & $\mathrm{I} / \mathrm{R}+\mathrm{A}+\mathrm{F}$ \\
\hline $\mathrm{SOD}, \mathrm{U} / \mathrm{mg}$ protein & $160.8 \pm 40.63$ & $87.8 \pm 26.44^{\mathrm{a}}$ & $122.3 \pm 21.08^{\mathrm{a}, \mathrm{b}}$ & $124.9 \pm 36.96^{\mathrm{a}, \mathrm{b}}$ & $126.0 \pm 27.81^{\mathrm{a}, \mathrm{b}}$ \\
\hline $\mathrm{MDA}, \mu \mathrm{mol} / \mathrm{mg}$ protein & $3.21 \pm 0.46$ & $8.99 \pm 1.82^{\mathrm{a}}$ & $4.74 \pm 0.67^{\mathrm{a}, \mathrm{b}}$ & $4.89 \pm 0.51^{\mathrm{a}, \mathrm{b}}$ & $4.49 \pm 0.64^{\mathrm{a}, \mathrm{b}}$ \\
\hline
\end{tabular}

$\mathrm{N}=12$, each group. ${ }^{\mathrm{a}} \mathrm{P}<0.05$ vs. sham group. ${ }^{\mathrm{b}} \mathrm{P}<0.05$ vs. I/R group. I/R, ischemia/reperfusion; $\mathrm{I} / \mathrm{R}+\mathrm{A}, \mathrm{I} / \mathrm{R}+$ atorvastatin; $\mathrm{I} / \mathrm{R}+\mathrm{F}, \mathrm{I} / \mathrm{R}+\mathrm{fasudil}$; $\mathrm{I} / \mathrm{R}+\mathrm{A}+\mathrm{F}, \mathrm{I} / \mathrm{R}+$ atorvastatin + fasudil; MDA, malondialdehyde; SOD, superoxide dismutase.

Committees of the Second Hospital of Shandong University, and all procedures were performed in accordance with the Institutional Animal Care and Use Committee and National Institutes of Health Guidelines.

Randomized assignment and treatment groups. A total of 60 rats were randomly and equally allocated into 5 groups ( $\mathrm{n=12/group):} \mathrm{i)} \mathrm{Sham} \mathrm{group;} \mathrm{ii)} \mathrm{I/R} \mathrm{group;} \mathrm{iii)} \mathrm{I/R} \mathrm{+} \mathrm{atorvastatin}$ group; iv) I/R + fasudil group; and v) I/R + atorvastatin + fasudil group. The sham group was used as a control and received a standard diet and water prior to the sham operation. The I/R group was used as a control and received a standard diet and water before I/R. The I/R + atorvastatin group was orally administered atorvastatin $(10 \mathrm{mg} / \mathrm{kg}$ body weight daily) for 2 weeks prior to I/R. The I/R + fasudil group was administered intravenously fasudil $(10 \mathrm{mg} / \mathrm{kg}$ body weight daily) for 2 weeks prior to I/R. The I/R + atorvastatin + fasudil group was administered the same doses of atorvastatin and fasudil prior to I/R.

In vivo heart $I / R$ injury model. Rats were anesthetized with sodium pentobarbital (50 mg/kg, intraperitoneally) and subjected to $30 \mathrm{~min}$ left anterior descending coronary artery (LAD) ligation using a 4-0 silk suture, followed by a $180 \mathrm{~min}$ reperfusion according to our previous published protocol (13). The sham control animals were subjected to the entire surgical procedure and silk suture was passed beneath the coronary artery, but the LAD coronary artery was not ligated. At the end of reperfusion, rats were exposed to overdoses of isoflurane for $\sim 10$ min and euthanatized by exsanguination.

Biochemical testing. Following reperfusion, arterial blood samples were collected and placed in heparinized centrifuge tubes, and were centrifuged at $1,500 \mathrm{x} \mathrm{g}$ for $20 \mathrm{~min}$ at $4^{\circ} \mathrm{C}$. The supernatant was collected and stored at $-80^{\circ} \mathrm{C}$. Serum IL- 6 and TNF- $\alpha$ activities were measured using Rat IL-6 and TNF- $\alpha$ ELISA kits (Bio-Swamp, Inc.; cat. no. RA20607; cat. no. RA20035, respectively), according to the manufacturer's protocols. Total nitric oxide (NO) production was determined by measuring the concentration of nitrate and nitrite, a stable metabolite of NO, by the modified Griess reaction method; the procedure involved the use of the Total NO kit (Beyotime Institute of Biotechnology), according to the manufacturer's protocols. Each sample supernatant $(100 \mu \mathrm{l})$ was reacted with Nitrate Reductase for $30 \mathrm{~min}$ and Griess reagent for $10 \mathrm{~min}$ at room temperature in the dark. Nitrite levels were determined by measuring absorbance at $540 \mathrm{~nm}$ using an OPTImax multiplate reader. The levels of nitrite were normalized to standard values.

Determination of myocardial infarct size. At the end of reperfusion, the coronary artery was re-occluded and Evans blue dye solution $(3 \mathrm{ml}, 2 \% \mathrm{wt} / \mathrm{vol})$ was injected into the left ventricle to determine between ischemic [area at risk (AAR), unstained] and non-ischemic myocardium (area not at risk, stained blue). The hearts were subsequently harvested, rinsed in normal saline and the atria, right ventricle and great vessels were removed. The heart was excised and cut into transverse slices (thickness, $1 \mathrm{~mm}$ ). The AAR was separated from the area not at risk and subsequently incubated with nitro-blue tetrazolium (NBT, $1 \% \mathrm{wt} / \mathrm{vol}, 15 \mathrm{~min}$ at $37^{\circ} \mathrm{C}$ ) to distinguish between ischemic (stained blue) and infarcted tissue (unstained). Then, the AAR and infarct size were calculated after weighing the respective tissue samples, and infarct size was expressed as the percentage of the AAR.

Evaluation of apoptosis in heart tissue sections. TUNEL was used to evaluate apoptotic activity following I/R injury. Heart sections were fixed in $4 \%$ paraformaldehyde for $24 \mathrm{~h}$ at room temperature and embedded in paraffin. Each section (thickness, $6 \mu \mathrm{m}$ ) was deparaffinized with xylene and rehydrated with serial changes ethanol (100, 95, 90, 80, 70, 60 and 50\%). 
TUNEL staining was performed using an in situ Cell Death Detection kit (Roche Diagnostics) according to the manufacturer's protocol. The TdT reaction was carried out for $1 \mathrm{~h}$ at $37^{\circ} \mathrm{C}$ in a humidified chamber, and then DAB chromogen (OriGene Technologies, Inc; cat. no. ZLI-9019) was applied. Hematoxylin was used as a counterstain. The results were viewed using a confocal FV 1000 SPD Laser Scanning microscope (Olympus Corporation; magnification, $\mathrm{x} 400$ ). TUNEL-positive cells were determined by randomly selecting 10 fields of view and were expressed as a percentage of normal nuclei.

Determination of superoxide dismutase (SOD) and malondialdehyde (MDA). A total of $20 \mathrm{mg}$ myocardial tissue was minced and homogenized in ice-cold RIPA buffer (Sigma-Aldrich; Merck KGaA). Homogenates were subject to centrifugation at $13,000 \mathrm{x} \mathrm{g}$ for $15 \mathrm{~min}$ at $4^{\circ} \mathrm{C}$ to obtain the supernatant as sample tissue for total protein preparation. The protein concentration was determined by bicinchoninic acid (BCA) assay kit (Beyotime Institute of Biotechnology). Myocardial tissue SOD activity and MDA content were assayed, according to the manufacturer's protocols (Jiancheng Biotech Ltd.; cat. no. A001-3-2; cat. no. A003-1-2). The results of SOD and MDA assays were expressed as units per $\mathrm{mg}$ of protein.

Western blot analysis for measurement of Rho-kinase activity and cleaved Caspase-3. Rho-kinase activity was assessed by examining the phosphorylation state of myosin phosphatase targeting subunit 1 (MYPT-1), a well-established Rho-kinase specific substrate (26). Western blotting was performed on heart tissue obtained from the AAR. Tissue proteins were extracted using the Membrane and Cytosol Protein Extraction kit (Beyotime Institute of Biotechnology; cat. no. P0033) and protein concentration was determined using the BCA assay kit (Beyotime Institute of Biotechnology) according to the manufacturer's instructions. After protein quantification, equal amounts of protein $(50 \mu \mathrm{g})$ were separated on $10 \%$ Tris-glycine SDS gel by electrophoresis and subsequently transferred to PVDF membranes. After blocking with 5\% BSA (Beyotime Institute of Biotechnology) in Tris-buffer for $1 \mathrm{~h}$ at room temperature, membranes were incubated overnight at $4^{\circ} \mathrm{C}$ with the primary antibodies: $\beta$-actin $(1: 1,000$; Santa Cruz Biotechnology, Inc.; cat. no. sc-69879), rabbit polyclonal MYPT-1 antibody (1:500; Bioworld Technology, Inc.; cat. no. BS8367), rabbit polyclonal phosphorylated (p)-MYPT-1 antibody (1:500; Bioworld Technology, Inc.; cat. no. BS64148) and rabbit monoclonal cleaved caspase-3 antibody (1:1,000; Cell Signaling Technology, Inc.; cat. no. 9664). Then, membranes were incubated at room temperature for $2 \mathrm{~h}$ with horseradish peroxidase-conjugated goat anti-rabbit secondary antibody (1:10,000; Santa Cruz Biotechnology, Inc.; cat. no. sc-2004). Immunoreactive bands were visualized with the SuperSignal West Pico enhanced chemoluminescence kit (Piece; Thermo Fisher Scientific, Inc.) according to the manufacturer's protocol. Band intensities were quantified using a densitometer analysis system Quantity One 4.6.6 (Bio-Rad Laboratories, Inc.).

Statistical analysis. Data are presented as the mean \pm standard deviation. Statistical analysis of the results was carried out
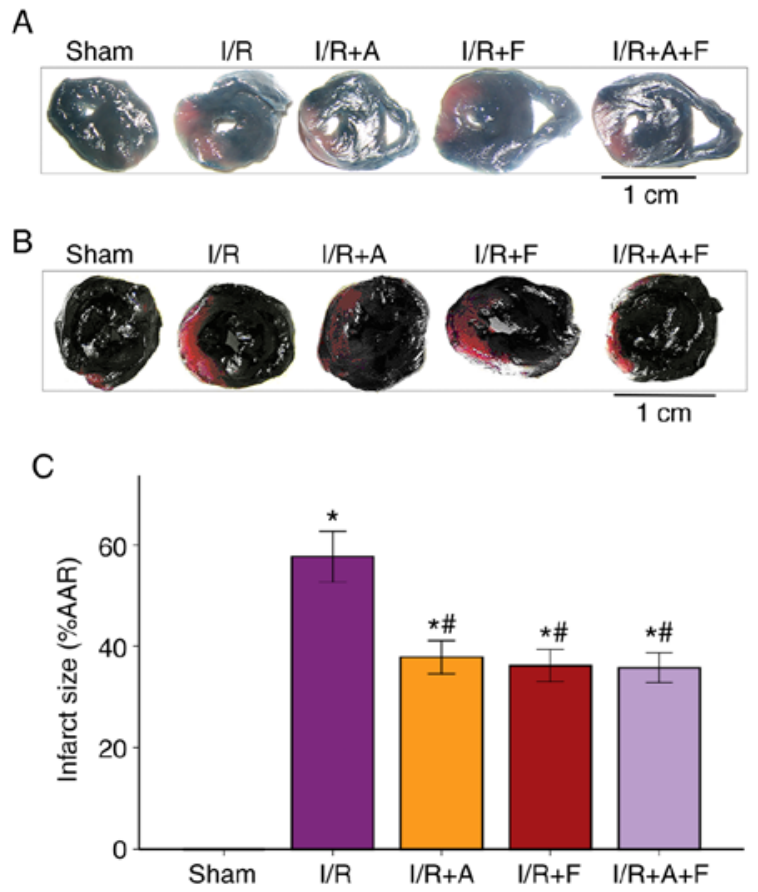

Figure 1. Changes of myocardial infarct size of different groups in rat hearts (A) Representative images of Evans blue-stained cross-sections of rat hearts. (B) Representative images of nitro-blue tetrazolium-stained cross-sections of rat hearts. Scale bar, $1 \mathrm{~cm}$. (C) Histograms of infarct size/AAR. ${ }^{*} \mathrm{P}<0.05$ vs. sham group. ${ }^{\#} \mathrm{P}<0.05$ vs. I/R group. I/R, ischemia/reperfusion; $\mathrm{I} / \mathrm{R}+\mathrm{A}, \mathrm{I} / \mathrm{R}+$ atorvastatin; $\mathrm{I} / \mathrm{R}+\mathrm{F}, \mathrm{I} / \mathrm{R}+$ fasudil; $\mathrm{I} / \mathrm{R}+\mathrm{A}+\mathrm{F}, \mathrm{I} / \mathrm{R}+$ atorvastatin + fasudil; AAR, area at risk

via one-way ANOVA followed by the Tukey's post-hoc test. $\mathrm{P}<0.05$ was considered to indicate a statistically significant difference. Statistical analyses were performed using SPSS 19.0 (SPSS, Inc.).

\section{Results}

Atorvastatin and fasudil reduce myocardial infarct size in heart $I / R$. It was identified that the average infarct size of the heart was $57.68 \pm 3.95 \%$ in the I/R group. Furthermore, administration of atorvastatin or fasudil caused a significant reduction in infarct size; the infarct size of the heart was $37.87 \pm 5.19$ and $36.21 \pm 5.01 \%$ in the $\mathrm{I} / \mathrm{R}+$ atorvastatin group and $\mathrm{I} / \mathrm{R}+$ fasudil group, respectively. Moreover, it was found that there were no significant differences between the $\mathrm{I} / \mathrm{R}+$ atorvastatin group, $\mathrm{I} / \mathrm{R}+$ fasudil group and $\mathrm{I} / \mathrm{R}+$ atorvastatin + fasudil group (Fig. 1). Therefore, the present results suggested that atorvastatin and fasudil reduced myocardial infarct size in rat heart I/R injury.

Atorvastatin and fasudil reduce cell apoptosis of the heart in $I / R$. Following examination of microscopy images, it was indicated that apoptotic cell nuclei were stained dark brown, while healthy myocardial cell nuclei were stained blue. The apoptotic rate was $2.04 \pm 1.34 \%$ in the sham group, while the number of TUNEL-positive cells was significantly increased in I/R group (36.83 $\pm 6.39 \%)$. In addition, it was demonstrated that the apoptotic rates were significantly reduced in the $I / R+$ atorvastatin, $I / R+$ fasudil and $\mathrm{I} / \mathrm{R}+$ atorvastatin + fasudil groups $(\mathrm{P}<0.05$ vs. I/R group $) ;$ 

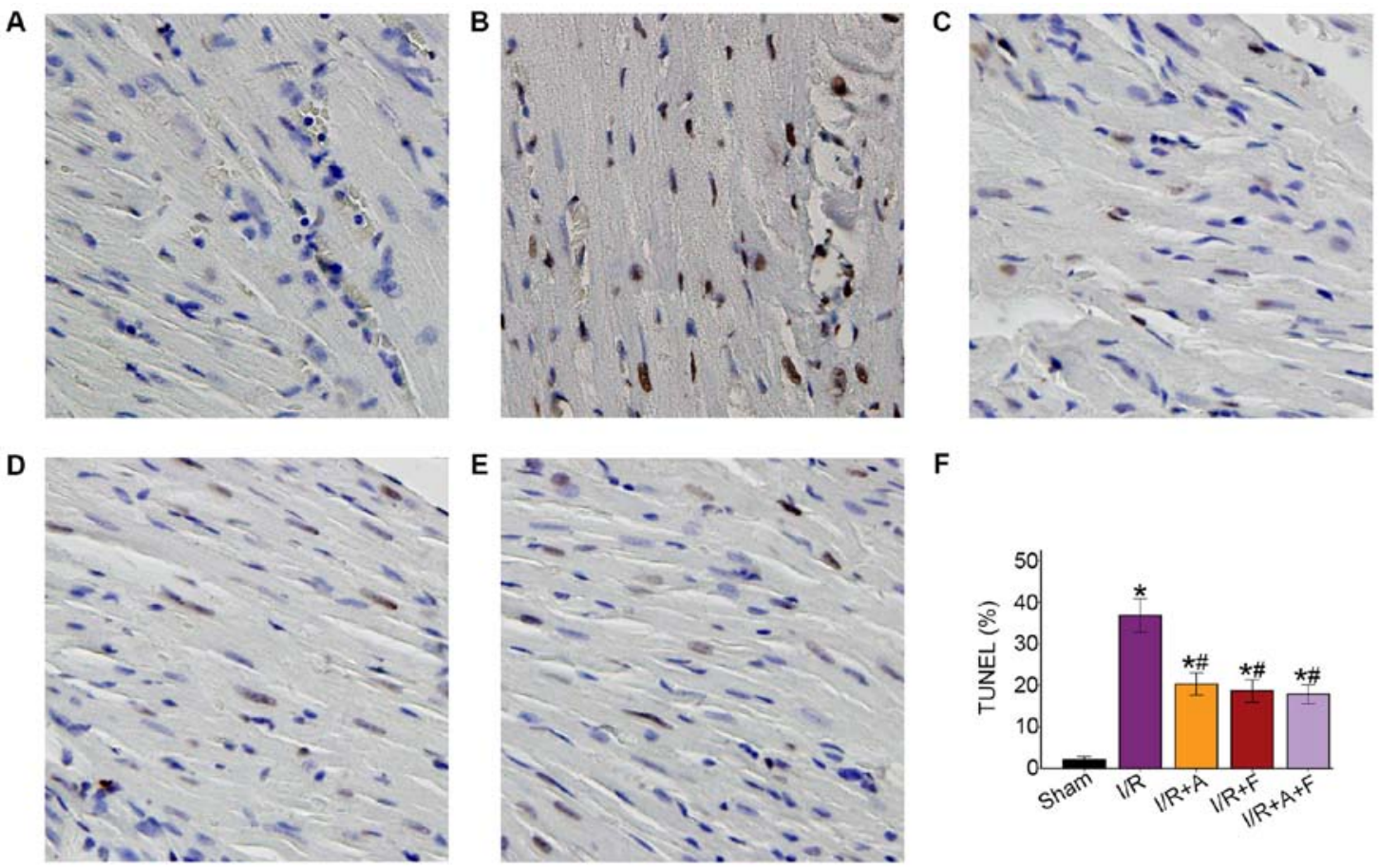

$\mathrm{F}$

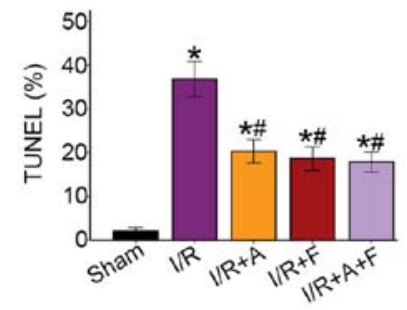

Figure 2. Representative photomicrographs of ventricular tissue stained for TUNEL to assess apoptosis. (magnification, $\mathrm{x} 400$ ). (A) Sham group. (B) I/R group. (C) $I / R+$ Atorvastatin group (D) $I / R+$ Fasudil group. (E) $I / R+$ Atorvastatin + Fasudil group. (F) Changes of TUNEL positive cells in different groups. In the I/R group, a large number of TUNEL-positive cells were observed. After administration of atorvastatin or fasudil, the TUNEL-positive cells were significantly reduced. " $\mathrm{P}<0.05$ vs. sham group. ${ }^{\#} \mathrm{P}<0.05$ vs. I/R group. I/R group. $\mathrm{I} / \mathrm{R}$, ischemia/reperfusion; $\mathrm{I} / \mathrm{R}+\mathrm{A}, \mathrm{I} / \mathrm{R}+$ atorvastatin; $\mathrm{I} / \mathrm{R}+\mathrm{F}, \mathrm{I} / \mathrm{R}+$ fasudil; $\mathrm{I} / \mathrm{R}+\mathrm{A}+\mathrm{F}$, $\mathrm{I} / \mathrm{R}+$ atorvastatin + fasudil.

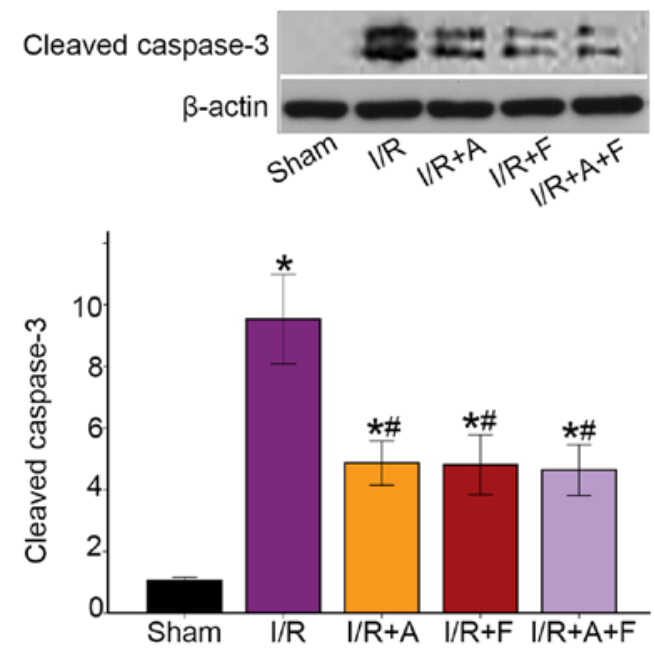

Figure 3. Atorvastatin and fasudil reduces cell apoptosis of the heart in I/R. Western blot analysis of cleaved caspase- 3 in different groups. ${ }^{*} \mathrm{P}<0.05$ vs. sham group. ${ }^{~} \mathrm{P}<0.05$ vs. I/R group. I/R group. $\mathrm{I} / \mathrm{R}$, ischemia/reperfusion; $\mathrm{I} / \mathrm{R}+\mathrm{A}, \mathrm{I} / \mathrm{R}+$ atorvastatin; $\mathrm{I} / \mathrm{R}+\mathrm{F}, \mathrm{I} / \mathrm{R}+$ fasudil $; \mathrm{I} / \mathrm{R}+\mathrm{A}+\mathrm{F}, \mathrm{I} / \mathrm{R}+$ atorvastatin + fasudil.

however, there were no significant differences among these three groups (Fig. 2).

Activation of caspase- 3 is a hallmark of apoptotic cell death, and caspase- 3 cleavage is indicative of its activation (27). The western blotting results identified that I/R caused a significant increase in the protein expression of cleaved capsase-3. Moreover, it was found that capsase- 3 activity was attenuated in the $\mathrm{I} / \mathrm{R}+$ atorvastatin, $\mathrm{I} / \mathrm{R}+$ fasudil and $\mathrm{I} / \mathrm{R}+$ atorvastatin + fasudil groups (Fig. 3). Thus, the results suggested that
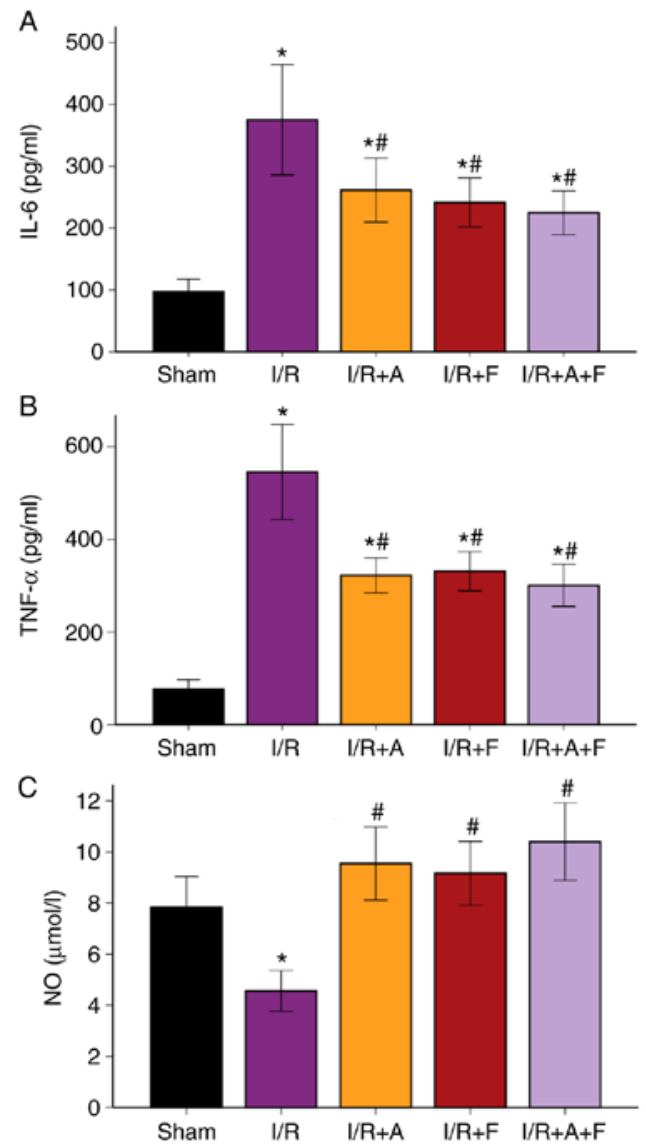

Figure 4. Changes in serum levels of (A) IL-6, (B) TNF- $\alpha$ and (C) NO in different groups. " $\mathrm{P}<0.05$ vs. sham group. ${ }^{*} \mathrm{P}<0.05$ vs. $\mathrm{I} / \mathrm{R}$ group. $\mathrm{I} / \mathrm{R}$ group. $\mathrm{I} / \mathrm{R}$, ischemia/reperfusion; $\mathrm{I} / \mathrm{R}+\mathrm{A}, \mathrm{I} / \mathrm{R}+$ atorvastatin; $\mathrm{I} / \mathrm{R}+\mathrm{F}, \mathrm{I} / \mathrm{R}+$ fasudil; $\mathrm{I} / \mathrm{R}+\mathrm{A}+\mathrm{F}, \mathrm{I} / \mathrm{R}+$ atorvastatin + fasudil; NO, nitric oxide; TNF, tumor necrosis factor; IL, interleukin. 

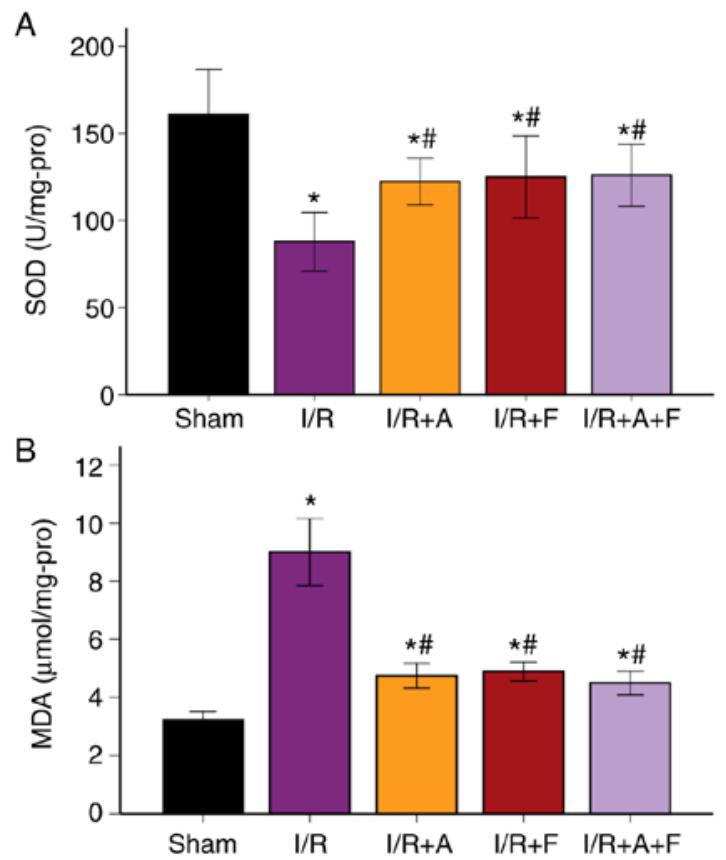

Figure 5. Changes in levels of (A) SOD and (B) MDA in different groups ${ }^{*} \mathrm{P}<0.05$ vs. sham group. ${ }^{\#} \mathrm{P}<0.05$ vs. I/R group. I/R group. I/R, ischemia/reperfusion; I/R + A, I/R + atorvastatin; I/R+F, I/R + fasudil $\mathrm{I} / \mathrm{R}+\mathrm{A}+\mathrm{F}, \mathrm{I} / \mathrm{R}+$ atorvastatin + fasudil; SOD, superoxide dismutase; MDA, malondialdehyde.

atorvastatin and fasudil reduced cell apoptosis in rat heart I/R injury.

Expression levels of plasma IL-6, TNF- $\alpha$ and NO in the different groups. Compared with the sham group, the serum concentrations of IL- 6 and TNF- $\alpha$ were significantly elevated in the $\mathrm{I} / \mathrm{R}$ group $(\mathrm{P}<0.05)$. Furthermore, these increases in IL- 6 and TNF- $\alpha$ levels were significantly suppressed in the $\mathrm{I} / \mathrm{R}+$ atorvastatin, $\mathrm{I} / \mathrm{R}+$ fasudil and $\mathrm{I} / \mathrm{R}+$ atorvastatin + fasudil groups $(\mathrm{P}<0.05 \mathrm{vs} . \mathrm{I} / \mathrm{R}$ group $) ;$ however, there were no significant differences among these three groups (Fig. 4A and B; Table I). It was also found that $\mathrm{NO}$ production in the I/R group was significantly decreased compared with the sham group $(\mathrm{P}<0.05)$. Moreover, when treated with atorvastatin, fasudil or their combination, NO production increased compared with the I/R group $(\mathrm{P}<0.05)$, but there were no significant differences among these three groups (Fig. 4C; Table I).

SOD activity and MDA levels in the different groups. It was indicated that MDA level was increased following I/R injury, while SOD decreased significantly ( $\mathrm{P}<0.05$ vs. sham group). In addition, when treated with atorvastatin, fasudil or their combination, the level of MDA was significantly suppressed, while SOD activity significantly increased $(\mathrm{P}<0.05$ vs. I/R group; Fig. 5A and B; Table II). Therefore, the results suggested that atorvastatin and fasudil antagonized oxidative stress induced by $\mathrm{I} / \mathrm{R}$ injury.

Rho-kinase activity in the different groups. Rho-kinase activity was assessed by examining the phosphorylation of MYPT-1 using western blot analysis and it was found that the phosphorylation of MYPT-1 was significantly increased
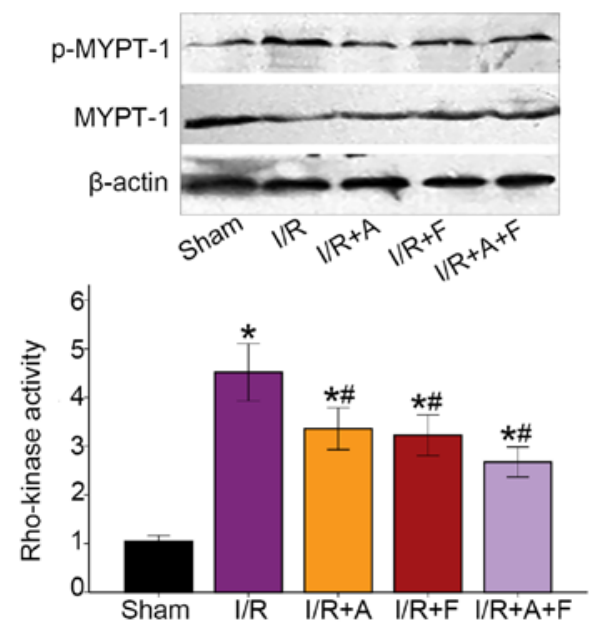

Figure 6. Western blot analysis of MYPT-1 phosphorylation in rat hearts of different groups. p-MYPT-1/MYPT-1 increased during the I/R groups, demonstrating Rho-kinase activity increased in the I/R. After administration of atorvastatin or fasudil, the expression of p-MYPT-1 was significantly attenuated. ${ }^{*} \mathrm{P}<0.05$ vs. sham group. ${ }^{*} \mathrm{P}<0.05$ vs. I/R group. I/R group. I/R, ischemia/reperfusion; I/R+A, I/R + atorvastatin; I/R+F, I/R + fasudil; I/R $+\mathrm{A}+\mathrm{F}, \mathrm{I} / \mathrm{R}+$ atorvastatin + fasudil; p, phosphorylated; MYPT-1, myosin phosphatase targeting subunit 1 .

during I/R protocol ( $\mathrm{P}<0.05$ vs. the sham group). Furthermore, atorvastatin, fasudil or their combination therapy resulted in significant reduction in $\mathrm{p}-\mathrm{MYPT}-1 / \mathrm{MYPT}-1$ ratio $(\mathrm{P}<0.05$ vs. I/R group; Fig. 6). Collectively, the results indicated that atorvastatin, similar to fasudil, inhibited Rho-kinase activity in heart I/R injury.

\section{Discussion}

In the present study, the effect of atorvastatin and fasudil was examined in heart I/R injury in the rat models. The major findings of the present in vivo study were as follows: i) Both fasudil and atorvastatin significantly attenuated myocardial $\mathrm{I} / \mathrm{R}$ injury in rat models, including myocardial infarct size, cardiomyocyte apoptosis, oxidative stress and inflammatory response; and ii) Rho-kinase inhibition was involved in the cardiovascular protective effects of atorvastatin in myocardial I/R.

Statins have been used to prevent coronary artery disease and stroke by lowering serum LDL cholesterol and inhibiting hepatic cholesterol biosynthesis (19). Previous studies have shown that statins have antiproliferative, antithrombotic, anti-inflammatory and anti-atherogenic effects, as well as their LDL cholesterol-lowering effects (24,28-30). Moreover, it has been revealed that atorvastatin provides cardioprotective effects against heart I/R injury via reducing myocardial infarct size and cardiomyocyte apoptosis (31-33). The average plasma half-life of atorvastatin is $\sim 14 \mathrm{~h}$, but the actual half-life of inhibition of HMG-COA reductase is 20-30 h due to the influence of its active metabolites (34). In the present study, the drugs were administered 2 weeks prior to surgery and atorvastatin was selected as the statin test agent. The present results indicated that pretreatment with atorvastatin attenuated myocardial infarction and myocardial apoptosis. Furthermore, it was found that atorvastatin resulted in a $44.8 \%$ reduction in 
apoptotic cardiomyocytes and a $34.3 \%$ reduction in myocardial infarct size, thus suggesting the cardiac protection provided by atorvastatin against heart I/R injury.

Oxidative stress, endothelial dysfunction and inflammation are among the most common mechanisms of heart $\mathrm{I} / \mathrm{R}$ injury $(35,36)$. It has been shown that statins increase endothelial NO production, which is impaired by $\mathrm{I} / \mathrm{R}$ (33). Furthermore, previous studies have reported that low-dose atorvastatin increases anti-inflammatory activity and increases NO concentration against $\mathrm{I} / \mathrm{R}$ injury in isolated hearts of rats (37). The present results indicated that $\mathrm{I} / \mathrm{R}$ increased levels of IL-6, TNF- $\alpha$ and MDA, while it decreased SOD and NO production. It was also observed that preventively administered atorvastatin attenuated the levels of IL-6, TNF- $\alpha$ and MDA, and upregulated SOD activity and NO production. Therefore, these results indicate that atorvastatin may attenuate $\mathrm{I} / \mathrm{R}$ heart injury, which may be mediated by reducing oxidative and inflammatory responses, and activating the NO pathway.

The Rho-kinase pathway, which serves an important role in a number of cardiovascular diseases, has been shown to be involved in heart I/R injury (5). In vitro studies reported that increased Rho-kinase activity downregulates NO production and that Rho-kinase inhibitors increase NO production $(38,39)$. It has also been revealed that atorvastatin prevents pulmonary vascular remodeling by inhibiting RhoA/Rho-kinase (40). Moreover, atorvastatin may upregulate NO levels via Rho-kinase signaling (41). Similar to our previous studies showing that the activation of Rho-kinase can be significantly upregulated by heart I/R injury $(13,42)$, the present results suggested that myocardial I/R caused a significant increase in Rho-kinase activity. Furthermore, Rho-kinase activity was assessed by examining the phosphorylation of MYPT-1 in the present study. It was found that $\mathrm{I} / \mathrm{R}$ heart injury resulted in a 4.5-fold increase in p-MYPT1 expression, thus indicating the activation of Rho-kinase following I/R injury. As fasudil is a specific and potent antagonist for Rho-kinase, it was identified that Rho-kinase activity was significantly decreased when treated with fasudil prior to I/R. The present study also examined whether Rho-kinase inhibition was involved in the cardiac protection of atorvastatin. A significant decrease in Rho-kinase activity was observed as a result of administration of atorvastatin and the combination of fasudil + atorvastatin. Therefore, it was speculated that inhibition of Rho-kinase may be involved in the cardioprotective effect of atorvastatin in heart I/R injury.

However, there are several limitations that require mentioning for the present study. Firstly, hemodynamic parameters were not determined and thus will be considered in future studies. In addition, it was found that atorvastatin lowered plasma cytokine levels and inflammation, but the results lack inflammatory infiltrate in myocardial tissues. Moreover, the underlying mechanism of Rho-kinase inhibition via atorvastatin in I/R injury remains to be fully elucidated. Future studies are required to assess whether atorvastatin inhibits Rho-kinase activity in a dose- and time-dependent manner. Furthermore, the present study only assessed the effect of atorvastatin on Rho-kinase inhibition by evaluating MYPT-1 phosphorylation, and therefore additional effectors of Rho-kinase are to be considered in future experiments. The aim of this study was also to identify the possible protective effects of atorvastatin, as continuous oral administration of atorvastatin could be beneficial for patients with acute myocardial infarction/percutaneous coronary intervention. Therefore, clinical trials registration will be considered in future research.

In conclusion, the present results indicated that atorvastatin may have a cardiovascular protective effect against I/R-induced injury, including inhibition of Rho-kinase activity. Thus, these findings may provide a novel approach for the process of statin administration and may provide new therapeutic strategies for myocardial I/R injury.

\section{Acknowledgements}

Not applicable.

\section{Funding}

The authors gratefully acknowledge research support provided by YouthFoundation of the National Natural Science Foundation of China (grant no. 81600284) and Shandong Key Research and Development Project (grant no. 2016GSF201196).

\section{Availability of data and materials}

The datasets used and/or analyzed during the current study are available from the corresponding author on reasonable request.

\section{Authors' contributions}

JZ designed the experiments and drafted the manuscript. CC and XL mainly performed the experiments and analyzed the data. SB and QL performed some of the experiments. All authors read and approved the final manuscript.

\section{Ethics approval and consent to participate}

The present study was approved by the Ethics Committees of the Second Hospital of Shandong University (Jinan, China).

\section{Patient consent for publication}

Not applicable.

\section{Competing interests}

The authors declare that they have no competing interests.

\section{References}

1. Laflamme MA and Murry CE: Heart regeneration. Nature 473: 326-335, 2011.

2. Szummer K, Jernberg T and Wallentin L: From early pharmacology to recent pharmacology interventions in acute coronary syndromes: JACC State-of-the-Art review. J Am Coll Cardiol 74: 1618-1636, 2019.

3. Hausenloy DJ and Yellon DM: Myocardial ischemia-reperfusion injury: A neglected therapeutic target. J Clin Invest 123: 92-100, 2013.

4. Fan ZX and Yang J: The role of microRNAs in regulating myocardial ischemia reperfusion injury. Saudi Med J 36: 787-793, 2015. 
5. Shimokawa H, Sunamura S and Satoh K: RhoA/Rho-kinase in the cardiovascular system. Circ Res 118: 352-366, 2016.

6. Zhou Q and Liao JK: Rho kinase: An important mediator of atherosclerosis and vascular disease. Curr Pharm Des 15: 3108-3115, 2009.

7. Zhang J, Xu DL, Liu XB, Bi SJ, Zhao T, Sui SJ, Ji XP and Lu QH Darapladib, a lipoprotein-associated phospholipase A2 inhibitor reduces rho kinase activity in atherosclerosis. Yonsei Med J 57: 321-327, 2016.

8. Patel P, Parikh M, Shah H and Gandhi T: Inhibition of RhoA/Rho kinase by ibuprofen exerts cardioprotective effect on isoproterenol induced myocardial infarction in rats. Eur J Pharmacol 791: 91-98, 2016.

9. Tanna AP and Johnson M: Rho Kinase inhibitors as a novel treatment for glaucoma and ocular hypertension. Ophthalmology 125 1741-1756, 2018.

10. Ocaranza MP, Moya J, Jalil JE, Lavandero S, Kalergis AM, Molina C, Gabrielli L, Godoy I, Córdova S, Castro P, et al: Rho-kinase pathway activation and apoptosis in circulating leucocytes in patients with heart failure with reduced ejection fraction. J Cell Mol Med 24: 1413-1427, 2020.

11. Fazakas C, Nagaraj C, Zabini D, Végh AG, Marsh LM, Wilhelm I, Krizbai IA, Olschewski H, Olschewski A and Bálint Z: Rho-kinase inhibition ameliorates Dasatinib-induced endothelial dysfunction and pulmonary hypertension. Front Physiol 9: 537, 2018.

12. Bao W, Hu E, Tao L, Boyce R, Mirabile R, Thudium DT, Ma XL, Willette RN and Yue TL: Inhibition of Rho-kinase protects the heart against ischemia/reperfusion injury. Cardiovasc Res 61: 548-558, 2004

13. Zhang J, Li XX, Bian HJ, Liu XB, Ji XP and Zhang Y: Inhibition of the activity of Rho-kinase reduces cardiomyocyte apoptosis in heart ischemia/reperfusion via suppressing JNK-mediated AIF translocation. Clin Chim Acta 401: 76-80, 2009

14. Zhang J, Bian HJ, Li XX, Liu XB, Sun JP, Li N, Zhang Y and Ji XP: ERK-MAPK signaling opposes rho-kinase to reduce cardiomyocyte apoptosis in heart ischemic preconditioning. Mol Med 16: 307-315, 2010.

15. Li Y, Zhu W, Tao J, Xin P, Liu M, Li J and Wei M: Fasudil protects the heart against ischemia-reperfusion injury by attenuating endoplasmic reticulum stress and modulating SERCA activity: The differential role for PI3K/Akt and JAK2/STAT3 signaling pathways. PLoS One 7: e48115, 2012.

16. Jiang ZH, Zhang TT and Zhang JF: Protective effects of fasudil hydrochloride post-conditioning on acute myocardial ischemia/reperfusion injury in rats. Cardiol J 20: 197-202, 2013.

17. Zhang YS, Tang LJ, Tu H, Wang SJ, Liu B, Zhang XJ, Li NS, Luo XJ and Peng J: Fasudil ameliorates the ischemia/reperfusion oxidative injury in rat hearts through suppression of myosin regulatory light chain/NADPH oxidase 2 pathway. Eur J Pharmacol 822: 1-2, 2018.

18. Huang YY, Wu JM, Su T, Zhang SY and Lin XJ: Fasudil, a rho-kinase inhibitor, exerts cardioprotective function in animal models of myocardial ischemia/reperfusion injury: A meta-analysis and review of preclinical evidence and Possible Mechanisms. Front Pharmacol 9: 1083, 2018.

19. Sirtori CR: The pharmacology of statins. Pharmacol Res 88: 1-3, 2014.

20. Istvan ES and Deisenhofer J: Structural mechanism for statin inhibition of HMG-CoA reductase. Science 292: 1160-1164, 2001.

21. Oesterle A, Laufs U and Liao JK: Pleiotropic effects of statins on the cardiovascular system. Circ Res 120: 229-243, 2017.

22. Tramontano AF, O'Leary J, Black AD, Muniyappa R, Cutaia MV and El-Sherif N: Statin decreases endothelial microparticle release from human coronary artery endothelial cells: Implication for the Rho-kinase pathway. Biochem Biophys Res Commun 320: 34-38, 2004

23. Yamanouchi D, Banno H, Nakayama M, Sugimoto M, Fujita $H$, Kobayashi M, Kuwano $\mathrm{H}$ and Komori K: Hydrophilic statin suppresses vein graft intimal hyperplasia via endothelial cell-tropic Rho-kinase inhibition. J Vasc Surg 42: 757-764, 2005.

24. McNeish AJ, Jimenez-Altayo F, Cottrell GS and Garland CJ: Statins and selective inhibition of Rho kinase protect small conductance calcium-activated potassium channel function $(\mathrm{K}(\mathrm{Ca}) 2.3)$ in cerebral arteries. PLoS One 7: e46735, 2012.
25. Ma MM, Li SY, Wang M and Guan YY: Simvastatin attenuated cerebrovascular cell proliferation in the development of hypertension through Rho/Rho-kinase pathway. J Cardiovase Pharmacol 59: 576-582, 2012

26. Narumiya $S$ and Thumkeo D: Rho signaling research: History, current status and future directions. FEBS Lett 592: 1763-1776, 2018.

27. Nagata S: Apoptosis and clearance of apoptotic cells. Annu Rev Immunol 36: 489-517, 2018

28. Li M, Liu Y, Dutt P, Fanburg BL and Toksoz D: Inhibition of serotonin-induced mitogenesis, migration, and ERK MAPK nuclear translocation in vascular smooth muscle cells by atorvastatin. Am J Physiol Lung Cell Mol Physiol 293: L463-L471, 2007.

29. Ridker PM, Danielson E, Fonseca FA, Genest J Gotto AM Jr, Kastelein JJ, Koenig W, Libby P, Lorenzatti AJ, MacFadyen JG, et al: Rosuvastatin to prevent vascular events in men and women with elevated C-reactive protein. N Engl J Med 359: 2195-2207, 2008

30. Rawlings R, Nohria A, Liu PY, Donnelly J, Creager MA, Ganz P, Selwyn A and Liao JK: Comparison of effects of rosuvastatin $(10 \mathrm{mg})$ versus atorvastatin $(40 \mathrm{mg})$ on rho kinase activity in caucasian men with a previous atherosclerotic event. Am J Cardiol 103: 437-441, 2009.

31. Sun T, Zhang HJ, Krittanawong C, Wang S, Tao Y, Li Z, Yin Q, Zhang D, Wang Q, Huang J, Zhang J, Li Z and Cheng Y: Acute atorvastatin treatment restores the cardioprotective effects of ischemic postconditioning in hyperlipidemic rats. Oncotarget 8 : 55187-55193, 2017

32. El Desoky ES, Hassan AKM, Salem SY, Fadil SA and Taha AF: Cardioprotective effect of atorvastatin alone or in combination with remote ischemic preconditioning on the biochemical changes induced by ischemic/reperfusion injury in a mutual prospective study with a clinical and experimental animal arm. Int J Cardiol 222: 866-873, 2016

33. Rohilla A, Ahmad A, Khan MU and Khanam R: A comparative study on the cardioprotective potential of atorvastatin and simvastatin in hyperhomocysteinemic rat hearts. Eur J Pharmacol 764: 48-54, 2015

34. Ye YC, Zhao XL and Zhang SY: Use of atorvastatin in lipid disorders and cardiovascular disease in Chinese patients. Chin Med J 128: 259-266, 2015.

35. Sydow K and Munzel T: ADMA and oxidative stress. Atheroscler Suppl 4: 41-51, 2003

36. Chatterjee A, Black SM and Catravas JD: Endothelial nitric oxide (NO) and its pathophysiologic regulation. Vascular Pharmacol 49: 134-140, 2008.

37. Lunder M,Ziberna L, Janić M, Jerin A, Skitek M, Sabovič M and Drevenšek G: Low-dose atorvastatin, losartan, and particularly their combination, provide cardiovascular protection in isolated rat heart and aorta. Heart Vessels 28: 246-254, 2013.

38. Takemoto M, Sun J, Hiroki J, Shimokawa H and Liao JK: Rho-kinase mediates hypoxia-induced downregulation of endothelial nitric oxide synthase. Circulation 106: 57-62, 2002

39. Rikitake Y, Kim HH, Huang Z, Seto M, Yano K, Asano T, Moskowitz MA and Liao JK: Inhibition of Rho kinase (ROCK) leads to increased cerebral blood flow and stroke protection. Stroke 36: 2251-2257, 2005

40. Wang Q, Guo YZ, Zhang YT, Xue JJ, Chen ZC, Cheng SY, Ou MD, Cheng KL and Zeng WJ: The Effects and mechanism of atorvastatin on pulmonary hypertension due to left heart disease. PLoS One 11: e0157171, 2016.

41. An L, An S, Jia Z, Wang H, Yang Z, Xu C, Teng X, Wang J, Liu X, Cao Q and Wang S: Atorvastatin improves left ventricular remodeling and cardiac function in rats with congestive heart failure by inhibiting RhoA/Rho kinase-mediated endothelial nitric oxide synthase. Exp Ther Med 17: 960-966, 2019.

42. Zhang J, Liu XB, Cheng C, Xu DL, Lu QH and Ji XP: Rho-kinase inhibition is involved in the activation of PI3-kinase/Akt during ischemic-preconditioning-induced cardiomyocyte apoptosis. Int J Clin Exp Med 7: 4107-4114, 2014.

This work is licensed under a Creative Commons Attribution-NonCommercial-NoDerivatives 4.0 International (CC BY-NC-ND 4.0) License. 